BMJ Open

Diabetes

Research

\& Care

\title{
Effect of a 1-week, eucaloric, moderately high-fat diet on peripheral insulin sensitivity in healthy premenopausal women
}

\author{
Natalia M Branis, ${ }^{1,2}$ Marjan Etesami, ${ }^{1,3}$ Ryan W Walker, ${ }^{1,4}$ Evan S Berk, ${ }^{1,5}$ \\ Jeanine B Albu ${ }^{1,6}$
}

To cite: Branis NM,

Etesami M, Walker RW, et al. Effect of a 1-week, eucaloric, moderately high-fat diet on peripheral insulin sensitivity in healthy premenopausal women. BMJ Open Diabetes Research and Care 2015;3 e000100. doi:10.1136/ bmjdrc-2015-000100

Received 8 March 2015 Revised 12 May 2015 Accepted 20 May 2015

\section{(a) CrossMark}

For numbered affiliations see end of article.

Correspondence to Dr Jeanine B Albu; jba1@columbia.edu

\section{ABSTRACT}

Objectives: To determine whether a weightmaintaining, moderate $(50 \%)$ high-fat diet is deleterious to insulin sensitivity in healthy premenopausal women.

Design/setting/participants: 23 African-American and non-Hispanic white, healthy, overweight, and obese premenopausal women recruited in New York City, USA, fed either a eucaloric, 1-week long high-fat ( $50 \%$ of total Kcal from fat) diet or a eucaloric, 1-week long low-fat (30\% of total Kcal from fat) diet, assigned in a randomized crossover design.

Main outcome measures: Peripheral insulin sensitivity and metabolic flexibility during a euglycemic hyperinsulinemic $\left(80 \mathrm{mU} / \mathrm{m}^{2} / \mathrm{min}\right)$ clamp measured during the follicular phase of the menstrual cycle, at the end of each diet period.

Results: Peripheral insulin sensitivity (mg kg/fat-free mass $/ \mathrm{min}(\mu \mathrm{U} / \mathrm{mL}) \times 10^{-1}$ ) was not decreased after the high-fat diet vs the low-fat diet $(0.09 \pm 0.01$ vs 0.08 $\pm 0.01, p=0.09$, respectively) in the combined group of African-American and white women, with no significant diet by race interaction $(p=0.6)$. Metabolic flexibility (change in substrate utilization, $\triangle \mathrm{NPRQ}$, in response to insulin during the clamp) was similarly unaltered by the diet $(0.12 \pm 0.01$ vs $0.11, p=0.48$, for the high-fat diet vs the low-fat diet, respectively) in the combined group of women, with no significant diet by race interaction ( $p=0.9$ ). African-American women had a lower insulin clearance compared with the white women, regardless of the diet $(p<0.05)$.

Conclusions: We conclude that a short term (1 week), moderate $(50 \%)$, eucaloric high-fat diet does not lower peripheral insulin sensitivity in healthy, overweight and obese premenopausal women.

\section{INTRODUCTION}

The role of the macronutrient composition of the diet with regard to the carbohydrate-to-fat ratio in the treatment of obesity and diabetes prevention has been only partially elucidated. While a low-fat (LF) diet was the mainstay for the diabetes prevention program ${ }^{1}$ and is the basis for the 2010 Dietary Guidelines for

\section{Key messages}

- There is controversy over whether a eucaloric, moderately high-fat $(50 \%)$ diet vs a lower fat $(30 \%)$ diet induces insulin resistance in overweight and obese women; substituting fat for carbohydrates to a moderate degree $(50 \%$ vs $30 \%$ ) in a weight-maintaining diet is not deleterious for peripheral insulin action in healthy overweight and obese women, at least in the short term (1 week).

- Similarly, metabolic flexibility (the ability to suppress fat oxidation by insulin during a hyperinsulinemic clamp) is not affected by a higher $(50 \%)$ vs a lower fat $(30 \%)$ eucaloric diet in healthy overweight and obese women, at least in the short term (1 week).

- African-American women are more insulin resistant and have lower rates of postabsorptive fat oxidation than similar white women, as we have previously reported, but we did not find that a moderately higher fat diet $(50 \%)$ compared to a lower fat diet $(30 \%)$ adversely affects their peripheral insulin action or ability to suppress fat oxidation during a high-dose insulin clamp.

Americans, ${ }^{2}$ hypocaloric diets of both high-fat (HF) and LF compositions have been effective for weight loss. ${ }^{3}$ Epidemiologically, higher total fat intake was associated with higher rates of progression to type 2 diabetes in the San Luis Valley Diabetes study ${ }^{4}$; however, two other large population-based studies in women (Iowa Women's and Nurses' Health studies) did not replicate these findings. ${ }^{5} 6$

Whether increasing the fat-to-carbohydrate ratio of a eucaloric, weight-maintaining diet decreases insulin sensitivity is controversial, particularly in women. ${ }^{7-12}$ One study in women has shown a decrease in insulin sensitivity, measured by a frequently sampled intravenous glucose tolerance test (FSIVGTT), after 3 weeks of an HF diet compared to a LF diet in healthy premenopausal 
African-American and non-Hispanic (NH) white participants. ${ }^{13}$ However, other work has demonstrated that peripheral insulin sensitivity, measured by the euglycemic hyperinsulinemic clamp, does not decrease after eucaloric $\mathrm{HF}$ diets of various durations (6 days and up to 3 weeks) in lean or obese men ${ }^{7-10}$ or combined groups of lean men and women. ${ }^{11}{ }^{12}$ Metabolic flexibility (the ability to suppress fat oxidation during the euglycemic hyperinsulinemic clamp) has been closely associated with insulin sensitivity ${ }^{14} 15$ and decreased in response to a HF diet in men, ${ }^{89}$ yet this has never been studied in women.

Therefore, we aimed to determine whether insulin sensitivity measured during a euglycemic hyperinsulinemic clamp will be deleteriously affected by a 1 week, eucaloric HF ( $50 \%$ total Kcal from fat) diet in AfricanAmerican and non-Hispanic white, healthy, premenopausal, overweight and obese women. In addition, we determined the effect of the diets on metabolic flexibility during the clamps. We and others have previously reported lower peripheral insulin sensitivity ${ }^{16-20}$ differences in muscle adipose tissue distribution ${ }^{19}$ and lower systemic rates of fat oxidation in African-American vs non-Hispanic white women. ${ }^{15} 2122$ Therefore, we also examined any race differences in substrate utilization during the clamps.

\section{RESEARCH DESIGN AND METHODS}

\section{Subjects}

Twenty-three healthy premenopausal (25-45 years) overweight and obese (body mass index, BMI $25-40 \mathrm{~kg} / \mathrm{m}^{2}$ ) women (11 African-American and 12 non-Hispanic white) participated in the study. Participants were included if they reported all four grandparents to be of African or Caucasian descent, had regular menstrual cycles, and were without diabetes according to an oral glucose tolerance test ( $75 \mathrm{~g}$ glucose load). Self-reported use of any medications (including contraceptive pills), smoking within the past 6 months, and consumption of $>2$ oz. ethanol/day were exclusionary. All participants signed consent forms approved by the St. Luke's-Roosevelt Institute for Health Sciences Institutional Review Board.

\section{Study design}

In a randomized crossover design, participants consumed a LF (30\% fat, $50 \%$ carbohydrate and $20 \%$ protein) or a HF (50\% fat, $30 \%$ carbohydrate and $20 \%$ protein) weight-maintaining diet for seven consecutive days as per the protocol we had previously published. ${ }^{15}$ On the morning of day 8 after an overnight admission to the Clinical Research Center at St. Luke's-Roosevelt Hospital Center, insulin sensitivity and substrate utilization were measured before and during a euglycemic hyperinsulinemic clamp. There was a minimum 2-week washout period between diets. All measurements were conducted during the follicular phase of the menstrual cycle.

\section{Dietary protocol}

All study participants completed dietary surveys indicating foods they liked and disliked. Eucaloric, weightmaintaining diets were constructed from food items available commercially with known macronutrient and caloric composition. Food item caloric content and macronutrient composition were verified using Nutritionist IV (V.2.0, Nsquared Commuting Co, Salem, Oregon, USA). Total daily calories for weight maintenance were calculated based on resting metabolic rate measured by indirect calorimetry in a fasting state (Horizon metabolic Cart or V-Max29; Sensor Medics, Yorba Linda, California, USA) and multiplied by an activity factor (1.5). Diets were matched in distribution of fat calories with equal parts of saturated fat, monounsaturated fat and polyunsaturated fat. Participants were provided with a 7-day food supply to consume at home. Dietary compliance was assured through weight stability measurements and adjustments were planned for a weight change of more than $1 \mathrm{~kg}$.

\section{Insulin sensitivity}

Following an overnight fast, a three-hour euglycemic hyperinsulinemic clamp $\left(80 \mathrm{mU} / \mathrm{m}^{2} / \mathrm{min}\right)$ was performed. We used a high-dose insulin clamp to measure the effect of the diet on peripheral insulin sensitivity in African-American vs non-Hispanic white women as we sought differences between races as well. We, as others, have previously reported lower peripheral insulin sensitivity $^{16-20}$ in African-Americans vs non-Hispanic whites. Blood samples were collected at $10 \mathrm{~min}$ intervals during the postabsorptive and steady state of hyperinsulinemic euglycemic clamp, immediately centrifuged, aliquoted and frozen at $-70^{\circ} \mathrm{C}$. Insulin was measured by RIA (Linco Research, St. Charles, Missouri, USA), glucose was measured by the Beckman glucose analyzer (Beckman, Fullerton, California, USA) and nonesterified fatty acids (NEFA) were measured by the enzymatic colorimetric method (Wako Chemicals USA, Richmond, Virginia, USA). NEFA suppression was calculated as the difference between the NEFA levels at steady state and the postabsorptive NEFA levels divided by the postabsorptive NEFA levels times 100 (percentage). Insulin clearance was calculated according to DeFronzo $^{23}$ as the ratio of the difference in insulin concentration between the post-absorptive and steady states and the rate of insulin infusion during the clamp study, which was assumed to be $80 \mathrm{mU} / \mathrm{m}^{2} / \mathrm{min}$ for all participants. Insulin sensitivity was calculated as M/I using the glucose disposal rate $\mathrm{M}$ ( $\mathrm{mg} \mathrm{kg} /$ fat-free mass (FFM)/ min) and insulin concentration in the hyperinsulinemic steady state $\mathrm{I}(\mu \mathrm{U} / \mathrm{mL})$.

\section{Indirect calorimetry}

Oxygen consumption $\left(\mathrm{VO}_{2}\right)$ and carbon dioxide production $\left(\mathrm{VCO}_{2}\right)$ were measured using a ventilated hood in the postabsorptive and hyperinsulinemic steady states of the euglycemic clamp. In both states, the participants 
Table 1 Participants' characteristics

\begin{tabular}{lllr}
\hline Characteristics & All participants (23) & African-American (11) & Non-Hispanic white (12) \\
\hline Age (years) & $33.61 \pm 1.18$ & $32.27 \pm 1.80$ & $34.83 \pm 1.54$ \\
BMl $\left(\mathrm{kg} / \mathrm{m}^{2}\right)$ & $29.65 \pm 0.90$ & $28.80 \pm 1.12$ & $30.42 \pm 1.39$ \\
Percentage of fat ${ }^{*}$ & $42.21 \pm 1.72$ & $40.03 \pm 2.57$ & $44.22 \pm 2.24$ \\
FM $(\mathrm{kg})^{*}$ & $33.83 \pm 2.14$ & $31.32 \pm 2.98$ & $36.13 \pm 3.02$ \\
FFM (kg)* & $44.85 \pm 0.91$ & $45.39 \pm 1.02$ & $44.35 \pm 1.51$ \\
Fasting triglycerides (mmol/L) & $0.85 \pm 0.11$ & $0.82 \pm 0.16$ & $0.88 \pm 0.16$ \\
Fasting HDL (mmol/L) & $0.90 \pm 0.04$ & $0.90 \pm 0.07$ & $0.91 \pm 0.07$ \\
\hline
\end{tabular}

Data are mean \pm SEM.

There were no significant differences in subject characteristics between African-American and non-Hispanic white women ( $\mathrm{p}$ range $0.23-0.91)$.

${ }^{*}$ Determined by dual X-ray absorptiometry (DXA).

BMI, body mass index; HDL, high-density lipoprotein; FM, fat mass; FFM, fat-free mass.

were supine and awake. Substrate oxidation rates were calculated using Frayn's equations, ${ }^{24}$ and non-protein respiratory quotient (NPRQ) was calculated as a ratio of $\mathrm{VCO}_{2}$ to $\mathrm{VO}_{2}$. Metabolic flexibility was estimated as a change in NPRQ $(\triangle \mathrm{NPRQ})$ between the postabsorptive and hyperinsulinemic steady states.

\section{Statistics}

All data are reported as mean \pm SEM as noted. All variables were checked for normality of distribution; only

Table 2 Effect of diet on insulin sensitivity and substrate utilization (repeated measures)

\begin{tabular}{lccc}
\hline & \multicolumn{2}{c}{ All subjects (19) } & \\
\cline { 2 - 3 } & Low-fat diet & High-fat diet & p Values \\
\hline Postabsorptive & & & \\
$\quad$ Glucose & $5.23 \pm 0.07$ & $5.18 \pm 0.08$ & 0.49 \\
Insulin & $71.78 \pm 4.90$ & $71.22 \pm 5.53$ & 0.77 \\
NEFA & $0.55 \pm 0.03$ & $0.53 \pm 0.03$ & 0.47 \\
NPRQ* & $0.90 \pm 0.01$ & $0.87 \pm 0.01$ & 0.12 \\
Steady state & & & \\
$\quad$ Glucose & $5.73 \pm 0.08$ & $5.67 \pm 0.08$ & 0.52 \\
Insulin & $1331.78 \pm 54.58$ & $1362.86 \pm 73.12$ & 0.41 \\
NEFA & $0.04 \pm 0.00$ & $0.04 \pm 0.00$ & 0.79 \\
NPRQ* & $1.01 \pm 0.01$ & $0.99 \pm 0.01$ & 0.15 \\
Ins. clearance & $455.73 \pm 20.29$ & $453.15 \pm 24.69$ & 0.67 \\
GDR $\dagger$ & $14.09 \pm 0.78$ & $15.51 \pm 0.84$ & 0.02 \\
M/I & $0.08 \pm 0.01$ & $0.09 \pm 0.01$ & 0.09 \\
NEFA \% & $92.90 \pm 0.67$ & $92.66 \pm 0.73$ & 0.72 \\
supp. & & & \\
NNPRQ* & $0.11 \pm 0.01$ & $0.12 \pm 0.01$ & 0.48 \\
\hline Da & & &
\end{tabular}

Data are mean \pm SEM. Glucose in $\mathrm{mmol} / \mathrm{L}$; insulin in $\mathrm{pmol} / \mathrm{L}$; non-esterified fatty acids (NEFA) in $\mathrm{mmol} / \mathrm{L}$; non-protein respiratory quotient (NPRQ); insulin clearance (Ins. Clear. in $\mathrm{mL} \mathrm{m}^{2} / \mathrm{BSA} / \mathrm{min}$; glucose disposal rate (GDR) in $\mathrm{mg} \mathrm{kg} / \mathrm{FFM} / \mathrm{min}$; insulin sensitivity $(M / I)=G D R /$ steady-state insulin; \% of non-esterified fatty acids suppression (NEFA \%); metabolic flexibility ( $\triangle N P R Q$ ).

${ }^{*}$ Data shown for 15 participants (7 African-American and 8 non-Hispanic white women) who completed substrate utilization measures for the LF and HF diets (repeated measures). $\mathrm{t} p=0.02$ for the effect of diet on GDR (higher after the HF diet); other effects of diet were not significant ( $p$ range $0.09-0.79$ ). BSA, body surface area; GDR, glucose disposal rate; HF, high-fat diet; LF, low-fat diet; NPRQ, non-protein respiratory quotient; NEFA, non-esterified fatty acids. fasting triglycerides were $\log$ transformed for analyses $(\log 10)$. Statistical comparison of participant characteristics by race was performed using the independent $t$ test (table 1). Selection and confounding biases were controlled for by symmetrical case-crossover methodology with identical length of exposure to the LF and $\mathrm{HF}$ diets. While the participants were unaware of the diet composition, there was no allocation concealment from the investigators.

Analysis of variance and multivariate analysis of variance (ANOVA/MANOVA) were used to determine the effects of diet (LF vs HF, repeated measures, within effect) and to compute diet by race interactions (African-Americans vs non-Hispanic whites, between effect) from measures in the postabsorptive state and during the steady state of the clamp (glucose, NEFA and insulin levels, substrate utilization, NEFA suppression, insulin clearance, insulin sensitivity and metabolic flexibility), as shown in table 2 and figures 1A, B and 2A, B. Only data from women who completed either one of the dietary interventions (LF or HF diet) were used for analysis. A power analysis was performed for the effect of diet on peripheral insulin sensitivity, using initial pilot data (first seven participants of the study), which yielded a large effect size, Cohen's $d=0.81$ (M/I change between diets mean \pm SD, $0.014262 \pm 0.017618$ ) from which the required sample size for 2 tailed $\alpha=0.05$, power $=0.80$ was calculated to be $\mathrm{n}=15$.

A general linear model was used to determine whether there were any race differences in response to insulin during the euglycemic hyperinsulinemic clamps, for the LF and HF diets separately (figure 2A and B). Both differences by race and any interactions by race in the effect of insulin during the clamps were computed for the LF and HF diets separately. The difference by race in steady-state insulin levels was also determined after adjusting for the postabsorptive insulin level (as a covariate). No other covariates were included in the analyses.

A p value less than 0.05 was considered statistically significant. Statistical analysis was performed using Statistica (V.10.0, Tulsa, Oklahoma, USA). 
Figure 1 (A-B) Insulin sensitivity after 7 days of a eucaloric low-fat diet (30\%) or high-fat $(50 \%)$ diet in non-Hispanic white (panel a) and African-American (panel b) women.
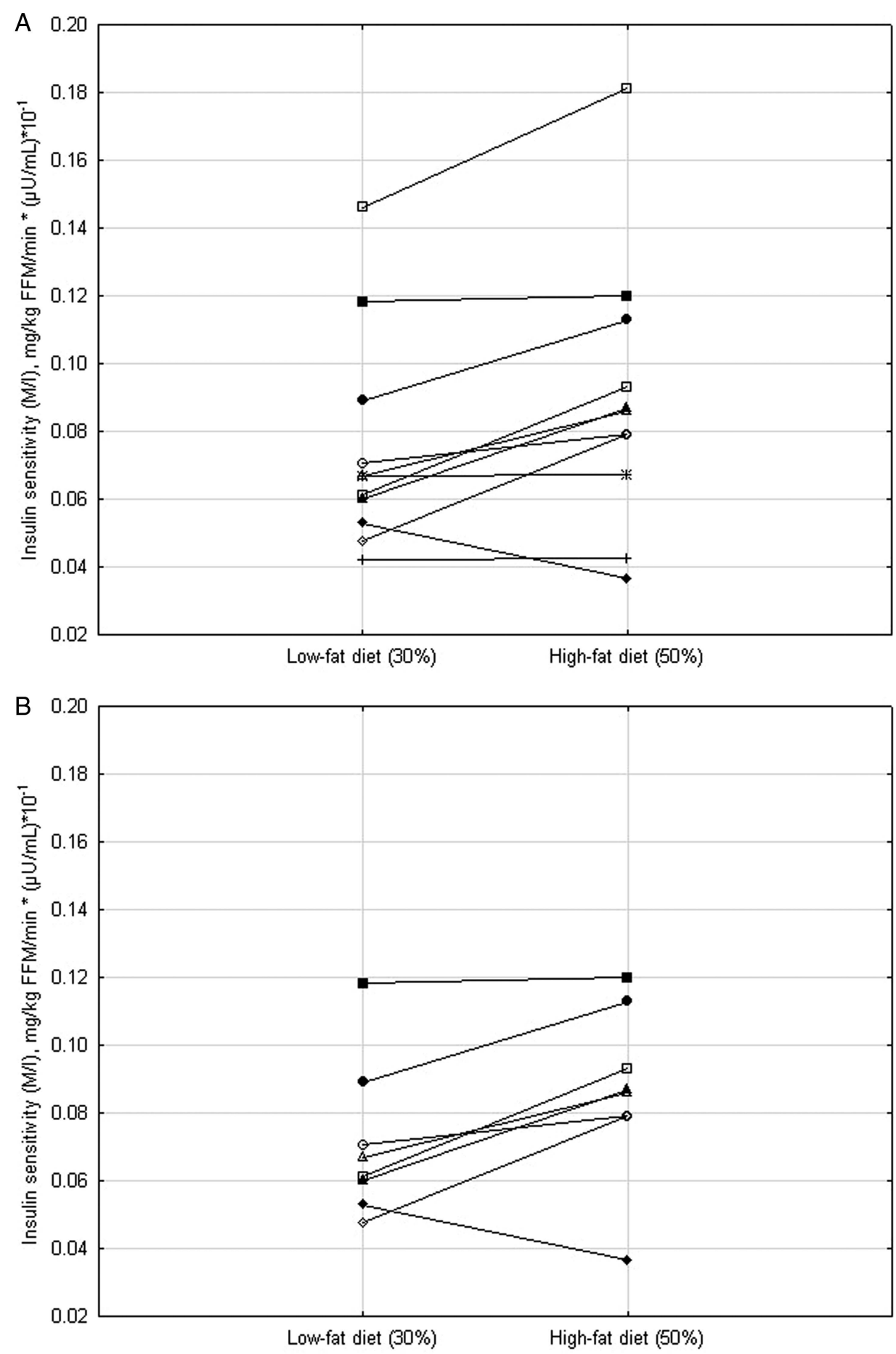

\section{RESULTS}

Participant characteristics are shown in table 1 . Twenty-three premenopausal (age $33.61 \pm 1.18$ years) overweight (BMI $29.65 \pm 0.90 \mathrm{~kg} / \mathrm{m}^{2}$ ) women participated in the study. Eight of 11 African-American and 11 out of 12 white women completed insulin sensitivity measurements after both the LF and HF diet periods (repeated measures). Additionally, one AfricanAmerican woman completed the studies only after the LF diet condition and three women (2 AfricanAmericans and 1 white) completed the studies only after the HF diet condition. For personal reasons, they did not participate in the second dietary period. There were no statistically significant differences in age, BMI, body composition measurements, fasting triglycerides and high-density lipoprotein (HDL)-cholesterol levels between the two races (table 1 ) or in the subgroups which had repeated measures (not shown).

For the 19 participants who had repeated measures, the effect of diet on insulin sensitivity and metabolic flexibility ( $\triangle \mathrm{NPRQ}$ during the clamp) are shown in table 2. There were no significant diet by race interactions on any 
Figure 2 (A-B) Differences in fat oxidation during the hyperinsulinemic euglycemic clamp between African-American and non-Hispanic white women after 7 days of a eucaloric low-fat diet $(30 \%)$ (A) OR after 7 days of a eucaloric high-fat diet (50\%) (B). Open circles, solid lines=African-American women. Open squares, dotted lines=non-Hispanic white women.
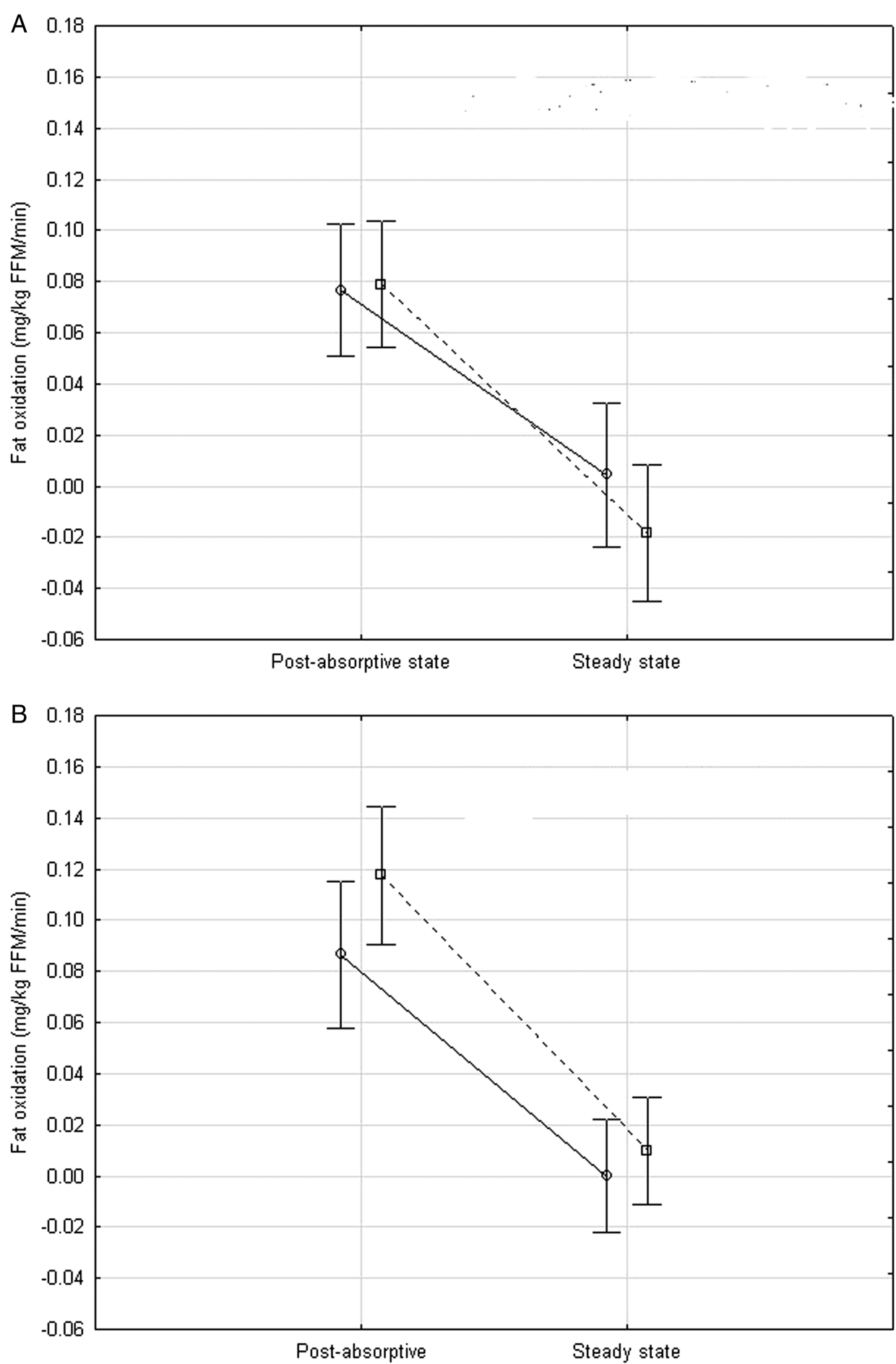

of the variables ( $\mathrm{p}$ range 0.31 to 1.0 ); thus, the main effects of diet are presented here. Insulin sensitivity computed as the glucose disposal rate per $\mathrm{kg}$ of FFM and divided by the steady-state insulin level $(\mathrm{M} / \mathrm{I})$ was not significantly decreased by the diet in the African-American $(0.06 \pm 0.01$ vs $0.07 \pm 0.01$, for LF vs HF diet, respectively, $\mathrm{p}=0.40)$ or in the white women $(0.09 \pm 0.01$ vs $0.10 \pm 0.01$, for LF vs HF diet, respectively, $\mathrm{p}=0.09)$. In most women, insulin sensitivity either remained unchanged or was higher after the HF compared to the LF diet (figure 1A and B). Similarly, metabolic flexibility $(\triangle \mathrm{NPRQ}$ during the clamp) was not significantly altered by the diet type (table 2).

Using data from all participants, the steady-state insulin levels during the clamp were higher in the AfricanAmerican vs non-Hispanic white women, after adjustment for the postabsorptive values, after the LF diet (1449.17 $\pm 40.34 \mathrm{pmol} / \mathrm{L}$ vs $1247.48 \pm 80.68 \mathrm{pmol} / \mathrm{L}, \mathrm{p}=0.02)$ or after the HF diet $(1490.98 \pm 59.59 \mathrm{pmol} / \mathrm{L}$ vs 1286.55 $\pm 103.75 \mathrm{pmol} / \mathrm{L}, \mathrm{p}=0.05)$. Thus, the calculated insulin clearance was lower in African-American vs white women, after the LF diet $\left(407.55 \pm 12.26 \mathrm{~mL} / \mathrm{m}^{2} / \mathrm{min}\right.$ vs 
$\left.489.60 \pm 30.19 \mathrm{~mL} / \mathrm{m}^{2} / \mathrm{min}, \mathrm{p}=0.03\right)$ or after the HF diet $\left(\left(397.02 \pm 14.27 \mathrm{~mL} / \mathrm{m}^{2} / \mathrm{min}\right.\right.$ vs $486.86 \pm 34.65 \mathrm{~mL} / \mathrm{m}^{2}$ ) $\min , \mathrm{p}=0.04)$. There were no other significant differences by race, after the LF diet ( $p$ range $0.91-1.0$ ) or after the HF diet ( $p$ range $0.14-0.9$ ).

Fat oxidation was significantly suppressed by insulin during the euglycemic clamp, for both AfricanAmerican and white women, after both the LF $(p<0.001)$ and HF $(\mathrm{p}<0.001)$ diets (figure $2 \mathrm{~A})$, with no significant insulin by race interaction (figure 2B) on either diet ( $p=0.27$ and $p=0.28$, respectively). $\triangle N P R Q$ that is, metabolic flexibility during the clamp, was not significantly different in African-American vs white women after the LF diet $(0.10 \pm 0.02$ vs $0.12 \pm 0.02$, respectively, $\mathrm{p}=0.59)$ or after the HF diet $(0.12 \pm 0.02$ vs $0.13 \pm 0.02, \mathrm{p}=0.58)$.

\section{CONCLUSIONS}

Our study did not show a decrease in peripheral insulin sensitivity in response to a short-term (1 week) eucaloric $50 \%$ HF diet compared to a $30 \%$ LF diet in healthy, overweight, and obese premenopausal African-American and non-Hispanic white women. Metabolic flexibility $(\triangle N P R Q)$ was similarly unaffected. The only significant race difference we found was the lower insulin clearance in AfricanAmerican vs white women, regardless of the diet.

Our results highlight the controversy surrounding the effect of a eucaloric increase in the fat content of a weight-maintaining diet on insulin sensitivity and metabolic flexibility, a precursor of insulin sensitivity. One other study, utilizing FSIVGTT to measure insulin sensitivity in premenopausal obese women showed a deterioration of insulin sensitivity after 3 weeks of a eucaloric HF diet vs a eucaloric LF diet, ${ }^{13}$ whereas other studies, in agreement with our results, have used a euglycemic hyperinsulinemic clamp to assess insulin sensitivity, which is the 'gold standard' for this outcome. A eucaloric HF diet consumed over a period of $\sim 3$ weeks did not alter insulin sensitivity in mixed groups of lean men and women, ${ }^{11}{ }^{12}$ and similar results were demonstrated in lean men after just 6 days, ${ }^{7}$ and in lean and overweight men after 3 weeks, ${ }^{8}{ }^{10}$ of a eucaloric HF diet. Thus, diet duration does not seem to account for the discrepancy between our results and other work in a similar population. ${ }^{13}$ Hepatic insulin sensitivity remained unchanged in two studies with a similar HF diet as used by us, ${ }^{10}$ but was shown to decrease in lean men after 11 days of an $83 \%$ HF diet. $^{9}$ FSIVGTT does not differentiate between hepatic and peripheral insulin $\times$ sensitivity. Different effects of a HF diet on hepatic vs peripheral insulin sensitivity may to some extent account for the difference in results noted by us. ${ }^{13}$ Other factors playing a role may be the account of the menstrual cycle stage when insulin sensitivity was measured, ${ }^{13} 25$ and the differences in the amounts of saturated fat employed. ${ }^{13}$

We also found that the metabolic flexibility, measured as a suppression of fat oxidation during the hyperinsulinemic $\left(80 \mathrm{mU} / \mathrm{m}^{2} / \mathrm{min}\right)$ euglycemic clamp $(\Delta \mathrm{NPRQ}),{ }^{14}$ was not affected by the 1 week of a eucaloric $50 \% \mathrm{HF}$ diet in our women. The effect of a eucaloric HF diet on $\triangle \mathrm{NPRQ}$ has been studied in men, yet the results are inconclusive. In lean men, $\triangle \mathrm{NPRQ}$ was not decreased in response to an HF $(75 \%)$ diet compared to a similar LF (35\%) diet, after 6 days, ${ }^{7}$ or 3 weeks, ${ }^{10}$ but was decreased after 11 days of a HF $(83 \%)$ diet. $^{9}$ In overweight men, $\triangle$ NPRQ decreased after 3 weeks of an HF $(55 \%)$ diet. $^{8}$ No similar studies are available in women. A certain threshold in the fat/carbohydrate ratio of the diet and the effect on hepatic insulin sensitivity ${ }^{26}$ may modulate the degree of fat oxidation suppression by insulin after a eucaloric HF diet. Hepatic insulin sensitivity and its relationship to metabolic flexibility was not evaluated in our study and needs to be investigated further. Some of the findings in the present study, specifically a lack of differential effect by race, may be due to a lack of power secondary to a small sample size. Furthermore, 1 week of a eucaloric $50 \%$ HF diet may have different effects in other populations, with different genetic susceptibility. ${ }^{27} 28$

Finally, we previously reported lower rates of postabsorptive fat oxidation in response to an HF diet and lack of fat oxidation suppression by insulin during a pancreatic clamp in African-American vs white women. ${ }^{15}$ In this study, we observed similar trends for the postabsorptive fat oxidation values, but the higher dose of insulin during the clamp similarly suppressed fat oxidation in the two races, in agreement with a recent report. ${ }^{29} \mathrm{We}$ also found lower insulin clearance in the AfricanAmerican women compared to the white women, in contrast to one, ${ }^{30}$ but in agreement with another study in adult women. ${ }^{31}$ The lower insulin clearance could be contributing to unmeasured postprandial hyperinsulinemia, which may partly explain the numerous reports of lower fat oxidation rates in African-Americans without diabetes compared to other white populations. ${ }^{21} 223233$

In conclusion, peripheral insulin sensitivity was not deleteriously affected by 1 week of a eucaloric HF diet (50\% of total Kcal from fat), compared to a LF (30\% of total Kcal from fat) diet, in healthy, premenopausal, overweight and obese African-American and non-Hispanic white women. Our findings need to be verified with regard to the effect on hepatic insulin response and more importantly in other susceptible populations.

\section{Author affiliations}

${ }^{1}$ New York Obesity Research Center, St. Luke's Roosevelt Hospital Center, New York, New York, USA

${ }^{2}$ Division of Endocrinology, Diabetes and Metabolism, University of Rochester School of Medicine and Dentistry, Rochester, New York, USA

${ }^{3}$ Department of Medicine, Palomar Medical Center, Escondido, California, USA ${ }^{4}$ The Charles Bronfman Institute for Personalized Medicine, Icahn School of Medicine at Mount Sinai, New York, New York, USA

${ }^{5}$ Nutrition Performance Unit, Glaxo Smith Kline, Parsippany, New Jersey, USA ${ }^{6}$ Division of Endocrinology, Diabetes and Nutrition, Mount Sinai St Luke's and Mount Sinai Roosevelt Hospitals, Icahn School of Medicine at Mount Sinai, New York, New York, USA

Acknowledgements The authors would like to acknowledge Allan Geliebter, $\mathrm{PhD}$, New York Obesity Research Center, for assistance and review of the statistical analyses. 
Contributors NMB analyzed the data, and wrote and prepared the manuscript for publication. ME analyzed the data and reviewed the manuscript. RWW collected the data, and reviewed and critiqued the manuscript. ESB designed the study, collected and analyzed the data, and reviewed and critiqued the manuscript. JBA designed the study, collected and analyzed the data, and wrote and prepared the manuscript for publication. She is also the guarantor of this work.

Funding This work was supported by the following grants: NIH R21DK71171, New York Obesity Research Center Grant P30DK26687, CTSA M01RR00645, DERC P30DK63608 and American Diabetes Association Grant 1-10-CT-01.

Competing interests JBA is an Associate Editor for Open BMJ DRC. She is also a reviewer of grants, abstracts and papers for the American Diabetes Association and its journals. JBA reports research grants funding from Weight Watchers, Eli Lilly, Roche, Takeda, Merck and Novo Nordisk, outside the submitted work. ESB is a current employee of GlaxoSmithKline. No other potential duality or conflicts of interest were reported relevant to this article.

Ethics approval St. Luke's-Roosevelt Institute for Health Sciences Institutional Review Board.

Provenance and peer review Not commissioned; externally peer reviewed.

Data sharing statement Methodology details information from this study is available through consultation with the corresponding author.

Open Access This is an Open Access article distributed in accordance with the terms of the Creative Commons Attribution (CC BY 4.0) license, which permits others to distribute, remix, adapt and build upon this work, for commercial use, provided the original work is properly cited. See: http:// creativecommons.org/licenses/by/4.0/

\section{REFERENCES}

1. Knowler WC, Barrett-Connor E, Fowler SE, et al. Reduction in the incidence of type 2 diabetes with lifestyle intervention or metformin. N Engl J Med 2002;346:393-403.

2. Dietary Guidelines for Americans. U.S. Department of Agriculture U.S. Department of Health and Human Services, 2010

3. Hite AH, Berkowitz VG, Berkowitz K. Low-carbohydrate diet review: shifting the paradigm. Nutr Clin Pract 2011;26:300-8.

4. Marshall JA, Hoag S, Shetterly S, et al. Dietary fat predicts conversion from impaired glucose tolerance to NIDDM. The San Luis Valley Diabetes Study. Diabetes Care 1994;17:50-6.

5. Meyer KA, Kushi LH, Jacobs DR Jr, et al. Dietary fat and incidence of type 2 diabetes in older lowa women. Diabetes Care 2001;24:1528-35.

6. Hu FB, Manson JE, Stampfer MJ, et al. Diet, lifestyle, and the risk of type 2 diabetes mellitus in women. N Engl J Med 2001;345:790-7.

7. Chokkalingam K, Jewell K, Norton L, et al. High-fat/low-carbohydrate diet reduces insulin-stimulated carbohydrate oxidation but stimulates nonoxidative glucose disposal in humans: An important role for skeletal muscle pyruvate dehydrogenase kinase 4. J Clin Endocrinol Metab 2007;92:284-92.

8. van Herpen NA, Schrauwen-Hinderling VB, Schaart G, et al. Three weeks on a high-fat diet increases intrahepatic lipid accumulation and decreases metabolic flexibility in healthy overweight men. J Clin Endocrinol Metab 2011;96:E691-695.

9. Bisschop PH, de Metz J, Ackermans MT, et al. Dietary fat content alters insulin-mediated glucose metabolism in healthy men. $A m \mathrm{~J}$ Clin Nutr 2011;73:554-9.

10. Cutler DL, Gray CG, Park SW, et al. Low-carbohydrate diet alters intracellular glucose metabolism but not overall glucose disposal in exercise-trained subjects. Metabolism 1995;44:1264-70.

11. Yost TJ, Jensen DR, Haugen BR, et al. Effect of dietary macronutrient composition on tissue-specific lipoprotein lipase activity and insulin action in normal-weight subjects. Am J Clin Nutr 1998;68:296-302.
12. Borkman M, Campbell LV, Chisholm DJ, et al. Comparison of the effects on insulin sensitivity of high carbohydrate and high fat diets in normal subjects. J Clin Endocrinol Metab 1991;72:432-7.

13. Lovejoy JC, Windhauser MM, Rood JC, et al. Effect of a controlled high-fat versus low-fat diet on insulin sensitivity and leptin levels in African-American and Caucasian women. Metabolism 1998;47:1520-4.

14. Galgani JE, Heilbronn LK, Azuma K, et al. Metabolic flexibility in response to glucose is not impaired in people with type 2 diabetes after controlling for glucose disposal rate. Diabetes 2008;57:841-5.

15. Berk ES, Kovera AJ, Boozer CN, et al. Metabolic inflexibility in substrate use is present in African-American but not Caucasian healthy, premenopausal, nondiabetic women. J Clin Endocrinol Metab 2006;91:4099-106.

16. Arslanian SA, Saad R, Lewy V, et al. Hyperinsulinemia in African-American children: decreased insulin clearance and increased insulin secretion and its relationship to insulin sensitivity. Diabetes 2002;51:3014-19.

17. Hannon TS, Bacha F, Lin Y, et al. Hyperinsulinemia in African-American adolescents compared with their American white peers despite similar insulin sensitivity: a reflection of upregulated beta-cell function? Diabetes Care 2008;31:1445-7.

18. Bacha F, Saad R, Gungor N, et al. Obesity, regional fat distribution, and syndrome $X$ in obese black versus white adolescents: race differential in diabetogenic and atherogenic risk factors. J Clin Endocrinol Metab 2008;88:2534-40.

19. Albu JB, Kovera AJ, Allen L, et al. Independent association of insulin resistance with larger amounts of intermuscular adipose tissue and a greater acute insulin response to glucose in African American than in white nondiabetic women. Am J Clin Nutr 2005;82:1210-17.

20. DeLany JP, Dube JJ, Standley RA, et al. Racial differences in peripheral insulin sensitivity and mitochondrial capacity in the absence of obesity. J Clin Endocrinol Metab 2014;99:4307-14.

21. Hickner R, Privette J, Mclver K, et al. Fatty acid oxidation in African-American and Caucasian women during physical activity. J Appl Physiol 2001;90:2319-24

22. Nicklas BJ, Berman DM, Davis DC, et al. Racial differences in metabolic predictors of obesity among postmenopausal women. Obes Res 1999;7:463-8.

23. DeFronzo RA, Tobin JD, Andres R. Glucose clamp technique: a method for quantifying insulin secretion and resistance. $A m \mathrm{~J}$ Physiol 1979;237:E214-223.

24. Frayn $\mathrm{KN}$. Calculation of substrate oxidation rates in vivo from gaseous exchange. J Appl Physiol 1983;55:628-34.

25. Yeung $\mathrm{EH}$, Zhang $\mathrm{C}$, Mumford SL, et al. Longitudinal study of insulin resistance and sex hormones over the menstrual cycle: the BioCycle Study. J Clin Endocrinol Metab 2010;95:5435-42.

26. Westerbacka J, Lammi K, Häkkinen AM, et al. Dietary fat content modifies liver fat in overweight nondiabetic subjects. J Clin Endocrinol Metab 2005;90:2804-9.

27. Ukropcova B, McNeil M, Sereda O, et al. Dynamic changes in fat oxidation in human primary myocytes mirror metabolic characteristics of the donor. J Clin Invest 2005;115:1934-41.

28. Ukropcova B, Sereda O, de Jonge L, et al. Family history of diabetes links impaired substrate switching and reduced mitochondrial content in skeletal muscle. Diabetes 2007;56:720-7.

29. Stull AJ, Galgani JE, Johnson WD, et al. The contribution of race and diabetes status to metabolic flexibility in humans. Metabolism 2010;59:1358-64

30. Lorenzo C, Hanley AJ, Wagenknecht LE, et al. Relationship of insulin sensitivity, insulin secretion, and adiposity with insulin clearance in a multiethnic population: the insulin Resistance Atherosclerosis study. Diabetes Care 2013;36:101-3.

31. Osei K, Schuster DP, Owusu SK, et al. Race and ethnicity determine serum insulin and C-peptide concentrations and hepatic insulin extraction and insulin clearance: comparative studies of three populations of West African ancestry and white Americans. Metabolism 1997;46:53-8.

32. Privette JD, Hickner RC, Macdonald KG, et al. Fatty acid oxidation by skeletal muscle homogenates from morbidly obese black and white American women. Metabolism 2003;52:735-8.

33. Chitwood L, Brown S, Lundy M, et al. Metabolic propensity towards obesity in black vs. white females: response during rest, exercise and recovery. Int J Obes 1996;20:455-62. 